\title{
A SET OF DEFINING RELATIONS FOR THE SIMPLE GROUP OF ORDER 1092*
}

\section{BY ABRAHAM SINKOV}

1. Introduction. This paper is essentially a continuation of a previous paper, by H. R. Brahana, entitled Certain perfect groups generated by two operators of orders two and three, $\dagger$ the major portion of which is devoted to the relations $S^{3}=T^{2}=(S T)^{7}=1$. The groups defined by these relations are all perfect, that is, they coincide with their commutator subgroups. Consequently, the order of the commutator of $S$ and $T$ is included as an additional defining relation. The purpose of this note is to study the case when this commutator is of order 7 and to show that the relations

$$
S^{3}=T^{2}=(S T)^{7}=\left(S^{-1} T^{-1} S T\right)^{7}=1
$$

completely define the simple group of order 1092. This result is then made use of to show that the non-alternating simple group of order 20,160 can not be generated by two operators of orders 2 and 3 .

2. Equivalent Generators. It is shown in Brahana's paper that the generators $S$ and $T$ may be replaced by a pair of equivalent generators $Q$ and $R$, in the sense that $\{S, T\}$ is the same as $\{Q, R\}$. The two sets of generators are connected by the relations

$$
S=R^{2} Q, \quad T=Q^{-1} R^{4} .
$$

In terms of $Q$ and $R$, the defining relations

$$
S^{3}=T^{2}=(S T)^{7}=\left(S^{-1} T^{-1} S T\right)^{7}=1
$$

become

$A: \quad R^{7}=Q^{7}=1, \quad Q^{-1} R^{4}=R^{3} Q, \quad R^{2} Q R^{-1}=Q^{-1} R Q$.

The method of determining the order of the group $G$ consists of an enumeration of the co-sets of $G$ as regards the cyclic group

* Presented to the Society, February 23, 1935.

$\dagger$ American Journal of Mathematics, vol. 50 (1928), p. 345 
generated by $R$; the proof of the completeness of this enumeration will follow from the representation of $R$ as a permutation on the symbols designating the various co-sets. This permutation shows how the co-sets are permuted under multiplication on the right by $R$.

The notation is the same as that used by Brahana. Thus, the co-set $e=Q^{2} R$ is made up of the seven operators $R^{\alpha} Q^{2} R$, $(\alpha=0,1, \cdots, 6)$, and $e_{i}$ is the co-set $R^{\alpha} Q^{2} R Q^{i},(i=1,2, \cdots, 6)$.

3. Definition of the Simple Group of Order 1092. It will be convenient to list a series of relations, obtainable from $A$, which greatly simplify the reduction to the final number of co-sets. The verification of these relations is, in some cases, lengthy but it is not deemed necessary to reproduce the manipulation here.
1. $Q^{\alpha} R^{3}=Q^{\alpha-1} R^{4} Q^{-1}$.
7. $Q^{4} R^{4}=R^{3} Q^{2} R^{4} Q^{2}$.
2. $Q^{-1} R^{4+\alpha}=R^{3} Q R^{\alpha}$.
8. $Q^{4} R^{6}=R^{5} Q^{2} R^{5} Q^{3}$.
3. $Q R^{2}=R Q R Q^{-1}$.
9. $Q^{5} R^{2}=R^{5} Q^{3} R Q^{3}$.
4. $Q R^{6}=R Q R^{4} Q$.
10. $Q^{5} R^{4}=R^{4} Q R^{4} Q^{2}$.
5. $Q^{4} R=R^{6} Q^{3} R^{6} Q^{3}$.
11. $Q^{5} R^{5}=R^{2} Q^{2} R^{2} Q^{2}$.
6. $Q^{4} R^{2}=R Q^{3} R^{5} Q^{2}$.
12. $Q^{5} R^{6}=R^{3} Q^{2} R Q^{6}$.

With the aid of these relations it follows that every combination of $Q$ and $R$ is reducible to an operator in some one of the co-sets listed below.

$$
\begin{aligned}
& a=1 \text {, } \\
& i=Q^{2} R^{6}, \\
& q=Q R Q^{2} R^{4}, \\
& b=Q R \text {, } \\
& j=Q^{3} R \text {, } \\
& r=Q R Q^{3} R^{4}, \\
& c=Q R^{4} \text {, } \\
& k=Q^{3} R^{2} \text {, } \\
& s=Q R^{4} Q^{3} R^{2}, \\
& d=Q R^{5}, \\
& l=Q^{3} R^{4}, \\
& t=Q R^{4} Q^{3} R^{6}, \\
& e=Q^{2} R \text {, } \\
& m=Q^{3} R^{5} \text {, } \\
& u=Q R^{4} Q^{5} R \text {, } \\
& f=Q^{2} R^{2} \text {, } \\
& n=Q^{3} R^{6} \text {, } \\
& v=Q R^{5} Q^{3} R^{4}, \\
& g=Q^{2} R^{4}, \\
& o=Q^{4} R^{5} \text {, } \\
& w=Q^{2} R^{4} Q^{3} R^{2} \text {, } \\
& h=Q^{2} R^{5} \text {, } \\
& p=Q^{5} R \text {, } \\
& x=Q R Q^{2} R^{4} Q^{3} R^{2} .
\end{aligned}
$$

Each of the above letters, with the exception of $v$ and $x$, takes on every subscript from 1 to 6 . The two co-sets $R^{\alpha} Q R^{5} Q^{3} R^{4}$ and $R^{\alpha} Q R Q^{2} R^{4} Q^{3} R^{2}$ are invariant under multiplication on the right by $Q$, so that neither $v$ nor $x$ may take any subscripts at all. 
The representation of $Q$ and $R$ as permutations of these 156 symbols is as follows:

$$
\begin{aligned}
Q= & \left(a a_{1} a_{2} a_{3} a_{4} a_{5} a_{6}\right)\left(b b_{1} b_{2} b_{3} b_{4} b_{5} b_{6}\right) \cdots \\
& \cdots\left(u u_{1} u_{2} u_{3} u_{4} u_{5} u_{6}\right)(v)\left(w w_{1} w_{2} w_{3} w_{4} w_{5} w_{6}\right)(x) . \\
R= & (a)\left(g_{4}\right)\left(a_{1} b b_{6} a_{6} c d c_{1}\right)\left(a_{2} e f c_{6} g h i\right) \\
& \left(a_{3} j k g_{6} l m n\right)\left(a_{4} n_{3} m_{2} l_{6} g_{2} o h_{3}\right)\left(a_{5} p j_{3} g_{1} c_{2} f_{2} e_{6}\right) \\
& \left(b_{1} f_{1} b_{5} d_{6} i_{6} p_{1} d_{1}\right)\left(b_{2} o_{6} e_{5} i_{5} q n_{4} j_{4}\right)\left(b_{3} m_{6} h_{2} q_{6} r j_{1} m_{3}\right) \\
& \left(b_{4} h_{6} n_{6} r_{6} p_{2} e_{1} k_{1}\right)\left(c_{3} h_{4} s p_{6} l_{5} k_{5} t\right)\left(c_{4} i_{3} q_{4} l_{4} l_{2} r_{2} p_{4}\right) \\
& \left(c_{5} u o_{2} l_{1} i_{1} s_{2} j_{6}\right)\left(d_{2} k_{2} o_{5} d_{5} r_{5} e_{3} q_{1}\right)\left(d_{3} d_{4} t_{3} r_{4} v q_{2} u_{4}\right) \\
& \left(e_{2} u_{3} o_{4} n_{5} k_{3} t_{4} e_{4}\right)\left(f_{3} s_{1} f_{6} j_{2} t_{6} u_{1} h_{1}\right)\left(f_{4} r_{1} j_{5} t_{5} m_{5} i_{4} o_{3}\right) \\
& \left(f_{5} k_{4} p_{3} m_{4} u_{2} h_{5} q_{5}\right)\left(g_{3} n_{1} w n_{2} g_{5} s_{5} s_{4}\right)\left(i_{2} p_{5} w_{5} u_{6} l_{3} t_{1} w_{2}\right) \\
& \left(k_{6} o_{1} w_{1} s_{6} m_{1} s_{3} w_{6}\right)\left(q_{3} u_{5} x t_{2} r_{3} w_{3} w_{4}\right) .
\end{aligned}
$$

These two substitutions then define a transitive group on 156 symbols. Since $G$ contains an operator of order 7 and is perfect, the following theorem results.

TheOREM 1. The relations

$$
S^{3}=T^{2}=(S T)^{7}=\left(S^{-1} T^{-1} S T\right)^{7}=1
$$

completely define the simple group of order 1092.

Since the subgroup $\left\{a, g_{4}\right\}$ is of order 14 , it may be used as the basis of a transitive representation of $G$ readily obtainable from the preceding representation and involving only 18 letters.

Combining this result with the definition given by Brahana for the same group, we see that two generators of the simple group of order 1092, whose orders are 2 and 3 and whose product is of order 7, may have a commutator of order either 6 or 7 .

4. Generation by Two Operators. Consider any group $G$ generated by two operators of orders 2 and 3 :

$$
S^{3}=T^{2}=1 .
$$

The commutator subgroup* $H$ of $G$ is generated by

* G. A. Miller, On the commutator groups, this Bulletin, vol. 4 (1897), p. 136. 


$$
\begin{array}{ll}
\sigma_{1}=S^{-1} T S T, & \sigma_{3}=T S^{-1} T S, \\
\sigma_{2}=T S T S^{-1}, & \sigma_{4}=S T S^{-1} T .
\end{array}
$$

But $\sigma_{1}=\sigma_{3}^{-1}, \sigma_{2}=\sigma_{4}^{-1}$. Therefore $H \equiv\left\{\sigma_{1}, \sigma_{2}\right\}$. Now, $\sigma_{1}$ and $\sigma_{2}$ are of the same order. Further

$$
\sigma_{1} \cdot \sigma_{2}=S^{-1} T S^{-1} T S^{-1}=S^{-1}\left(T S^{-1} T S\right) S,
$$

and is of the same order as $\sigma_{1}$. Therefore $H$ is generated by two operators of order $n$, whose product is of order $n$.

Returning to the groups defined by

$$
S^{3}=T^{2}=(S T)^{7}=1
$$

which are perfect, we see that they may also be generated by the two operators $\sigma_{1}$ and $\sigma_{2}$.

THEOREM 2. The simple group of order 1092 may be generated by two operators of order 6 whose product is of order 6 , or by two operators of order 7 whose product is of order 7 .

5. Non-alternating Simple Group of Order 20,160. Adding the results of this paper to those already obtained by Brahana, the groups defined by the relations

$$
S^{3}=T^{2}=(S T)^{7}=1
$$

are known for every order of $S^{-1} T^{-1} S T$ up to and including 7 . These results may be applied to the non-alternating simple group of order 20,160 as follows. The operators contained within this group* must be of order $2,3,4,5$, or 7 . If then, the group may be generated by two operators of orders 2 and 3 the product of these generators must be of order 7. But the order of their commutator could not exceed 7 , and a contradiction results.

THEOREM 3. It is not possible for two operators of orders 2 and 3 to generate the non-alternating simple group of order 20,160.

Washington, D.C.

* L. E. Dickson, Linear Groups with an Exposition of the Galois Field Theory, p. 259. 